Pacific Journal of Mathematic 


\section{COMPACTNESS IN ABELIAN TOPOLOGICAL GROUPS}

\section{RANGACHARI VENKATARAMAN}

If an abelian topological group $(G, t)$ satisfies Pontryagin duality, then the $t$-compact subsets and the weak compact subsets coincide. Hence if $t_{1}, t_{2}$ are group topologies on an abelian group $G$ such that $\left(G, t_{i}\right) i=1,2$ satisfy Pontryagin duality and have the same set of continuous characters, then $t_{1}=t_{2}$.

1. Introduction. Our object is to prove the following two theorems:

THEOREM 1.1. Let $(G, t)$ be an abelian topological group which satisfies Pontryagin duality. Let $w$ be the weak topology induced by the set $X$ of all its continuous characters. Then a subset $A$ of $G$ is t-compact iff it is w-compact.

THEOREM 1.2. Let $t_{1}, t_{2}$ be topologies on an abelian group $G$ such that $\left(G, t_{i}\right), i=1,2$, is a topological group that satisfies Pontryagin duality. If the sets of continuous characters of $\left(G, t_{1}\right)$ and $\left(G, t_{2}\right)$ coincide, then $t_{1}=t_{2}$.

Glicksberg ([5] Theorem 1.2 and Corollary 2.4) proves the above two results for the special case of locally compact abelian groups. Glicksberg's work has been generalized in different manners. One such is to start with a locally compact group $(G, t), G$ not necessarily abelian, consider the weak topology $w$ induced by the class of $t$ continuous irreducible unitary representations of $G$ and ask whether compactness with respect to $t$ and $w$ coincide. This corresponds to Theorem 1.1 above. Corresponding to Theorem 1.2 one can ask whether if $t_{1}, t_{2}$ are locally compact topologies on a not necessarily abelian group $G$ such that both $\left(G, t_{1}\right)$ and $\left(G, t_{2}\right)$ are topological groups and have the same set of continuous irreducible unitary representations, is $t_{1}=t_{2}$ ? These two questions were answered in the affirmative by Hughes ([3], Theorem 1, page 18, and Corollary 1.3, page 19). (See also [4] Theorems 1 and 2.) Another direction of generalization of Glicksberg's results is to regard the set $X$ of continuous characters of $G$ as $\operatorname{Hom}(G, T)$ where $T$ is the circle group and examine the following question. Let $G$ and $H$ be topological groups and $\operatorname{Hom}(G, H)$, the set of all continuous homomorphisms of $G$ into $H$. Do compactness with respect to the pointwise convergent topology and the compact-open topology on $\operatorname{Hom}(G, H)$ coincide? 
Corson and Glicksberg ([1] Theorem 1) asserted that if $G$ is such that the closure of every compactly generated subgroup is of second category in itself, this is indeed so. Most recently Namioka [10] has remarked that the proof of this assertion in [1] is incomplete. Using different methods, Namioka ([10], Theorem 3.3) proves the above result in the special case when $G$ is strongly countably compact. However the assertion of Corson and Glicksberg is proved by using an independent approach by Hughes ([3] Theorem 3). (See also [4] Theorem 4.)

Our objective in this paper is to examine Glicksberg's results in [5] referred to above for the case of abelian topological groups which satisfy Pontryagin duality and which are not necessarily locally compact abelian groups. That such groups exist is well known (cf. for instance, Kaplan [7] and Venkataraman [11]). The proofs in this direction of generalization are much simpler than those in the other directions mentioned above. Our proofs avoid measure theory and Fourier transforms and use only results on the topologies on function spaces. In the case of Theorem 1.1, our approach to the proof is the same as Hughes ([3] Theorem 3) but becomes different when we invoke the use of Bohr compactifications. The proof of Theorem 1.2 depeds upon a result of mine ([11] Theorem 5.3).

2. Proofs of Theorems 1.1 and 1.2. For notation and terminology relating to topological groups we follow Hewitt and Ross [2].

Let $T$ denote the circle group. A homomorphism of a group $G$ to $T$ is called a character of $G$. The set $X$ of all continuous characters of a topological group $G$ endowed with the compact-open topology and multiplication defined pointwise is a topological group, called the character group of $G$. $G$ is said to satisfy Pontryagin duality if the evaluation map $\tau_{G}: G \rightarrow \omega$ (where $\omega$ is the character group of $X$ ) defined by $\tau_{G}(x) \chi=\chi(x)$ for every $\chi \in X$ and $x \in G$ is a topological isomorphism.

Definition 2.1. Let $G$ be an abelian topological group, $X$ the character group of $G, W$ a limited neighbourhood of 1 in $T$, i.e. a symmetric connected neighbourhood of 1 which is contained in $\{z \in$ $T|| z-1 \mid \leqq \sqrt{2}\}$ (cf. Kaplan [7], page 650). Let $S \supset G$. By the $W$-character closure of $S$ (in symbols $W$-chr.cl. $S$ ) we mean

$$
\cap\left\{\chi^{-1}(W) \mid \chi \in X, \chi(S) \subset W\right\} \text {. }
$$

If $S=W$-chr. cl. $S$, we say that $S$ is $W$-character closed. 
Proposition 2.2. Let $G$ satisfy Pontryagin duality. Then the following properties hold:

$\left(P_{1}\right): G$ has sufficiently many continuous characters.

$\left(P_{2}\right)$ : Let $U$ be $W$-character closed subset of $G$ for some closed limited neighbourhood $W$ of 1 in $T$ such that for every compact subset $K$ of $G$ with $K$ containing the identity element e of $G, U \cap K$ is a neighbourhood of e relative to $K$. Then $U$ is a neighbourhood of $e$ in $G$.

$\left(P_{3}\right): G$ admits a family of $W$-character closed subsets as a neighbourhood base at e for some limited neighbourhood $W$ of 1 in $T$.

Proof. Any topological group that satisfies Pontryagin duality has sufficiently many continuous characters and hence $G$ satisfies $P_{1}$. That such a group satisfies the property $P_{2}$ is proved in Theorem 5.3 of Venkataraman [11]. We use the notations in the beginning of this section. By Proposition 2.9 of Kaplan [7], $\left\{P\left(K^{*}, W\right) \mid K^{*}\right.$ a compact subset of $X$ \} is a neighbourhood base at the identity element of $\omega$ where $W$ is (any) one limited neighbourhood of 1 in $T$ and $P\left(K^{*}, W\right)=\left\{f \in \omega \mid f\left(K^{*}\right) \subset W\right\}$. It is easy to see that for each subset $K^{*}$ of $X, P\left(K^{*}, W\right)$ is a $W$-character closed subset of $\omega$. Thus $\omega$ admits a neighbourhood base of $W$-character closed subsets at the identity element, i.e. $\omega$ satisfies property $P_{3}$. Since by hypothesis $G$ satisfies Pontryagin duality, $\tau_{G}: G \rightarrow \omega$ is a topological isomorphism. It follows that $G$ satisfies $P_{3}$. The proof of our proposition is complete.

For notation and terminology on topologies of function spaces we follow Kelley ([8], Chapter 7).

Proof of Theorem 1.1. Clearly any $t$-compact subset of $G$ is $w$ compact as $w$ is coarser than $t$. So let $A$ be a $w$-compact subset of $G$. As $G$ satisfies Pontryagin duality, the topology $t$ on $G$ is the compact open topology $\mathscr{C}$ when $G$ is regarded in the canonical manner as the character group of $X$. Also in this context, the weak topology $w$ on $G$ determined by $X$ is the topology $\mathscr{P}$ of pointwise convergence on $X$. Thus what we are required to prove is that if $A \subset G$ and $A$ is $\mathscr{P}$-compact, then $A$ is $\mathscr{C}$-compact. From the sixth of the six equivalent statements in Hughes' version of the Arzela-Ascoli Theorem (cf. Hughes [3], Proposition 15), it suffices to consider compact subset $D$ of $X$ and any sequence $\left\{f_{n}\right\}_{n}$ in $A$, and prove the following: $\left(^{*}\right)$ There is a subsequence $\left\{f_{n_{k}}\right\}_{k}$ of $\left\{f_{n}\right\}_{n}$ such that $\left\{\left.f_{n_{k}}\right|_{D}\right\}_{k}$ is equicontinuous.

Since $A$ is by hypothesis $\mathscr{P}$-compact, a fortiori $\left\{\left.f\right|_{D} \mid f \in A\right\}$, which we shall denote by $\left.A\right|_{D}$, is $\mathscr{P}$-compact. So by Theorem 5, Grothendieck [6], $\left.A\right|_{D}$ is sequentially compact. Therefore there exists a subsequence 
$\left\{f_{n_{k}}\right\}_{k}$ of $\left\{f_{n}\right\}_{n}$ such that $\left\{f_{n_{k}}\right\}_{k}$ is convergent pointwise on $D$ to some $f$ in $A$. Now since $G$ satisfies Pontryagin duality and any compact abelian group satisfies Pontryagin duality, $G$ can be regarded as the set of all continuous characters of $X$ and $b X$. Here $b X$ is the so called Bohr compactification of $X$, i.e. $b X$ is the compact character group of $(G, d)$ where $d$ is the discrete topology on $G$. Let $\rho$ be the canonical map of $X$ to $b X$ which identifies a continuous character of $(G, t)$ with a (continuous) character of $(G, d)$. It is easily verified that in our case $\rho$ is a continuous monomorphism of $X$ onto a dense subgroup of $b X$. Furthermore the topology on $G$ of pointwise convergence on $b X$ (equivalently $\rho(X)$ ) is the same as $\mathscr{P}$. Thus the subset $A \subset G$ we started with can be regarded as a $\mathscr{P}$-compact subset of continuous functions from $b X$ to $T$ and the subsequence $\left\{f_{n_{k}}\right\}_{k}$ of $\left\{f_{n}\right\}_{n}$ is pointwise convergent on $\rho(D)$ to $f$ in $A$. Let $F$ be the closed subgroup of $b X$ generated by $D$. The topology on $\left.A\right|_{F}$ of pointwise convergence on $b X$ is clearly compact as $A$ is $\mathscr{P}$-compact. This implies that the topology $\mathscr{P}_{1}$ on $\left.A\right|_{F}$ of pointwise convergence on $\rho(D)$ is compact. Furthermore it is $T_{2}$. For if $g,\left.h \in A\right|_{F}$ and $g \neq$ $h$, as $F$ is the closed subgroup generated by $\rho(D)$, there exists $\chi$ in $\rho(D)$ such that $g(\chi) \neq h(\chi)$. So $\rho(D)$ distinguishes members of $\left.A\right|_{F}$. So by Theorem 2 page 220 Kelley [8], the topology $\mathscr{P}_{1}$ is $T_{2}$. The topology $\mathscr{P}_{2}$ on $\left.A\right|_{F}$ of pointwise convergence on $F$ is also compact as $A$ is compact with respect to pointwise convergence on $b X$, and clearly $\mathscr{P}_{2}$ is coarser than $\mathscr{P}_{1}$. So $\mathscr{P}_{2}=\mathscr{P}_{1}$. Since the subsequence $\left\{f_{n_{k}}\right\}_{k}$ converges pointwise on $\rho(D)$ to $f$ it now follows that it converges pointwise on $F$ to $f$. As the topology on $T$ is given by an invariant metric, by Osgood's theorem (Theorem 9.5, Kelley, Namioka and coauthors [9]) the set of points of equicontinuity of $\left\{\left.f_{n_{k}}\right|_{F}\right\}_{k}$ is residual in $F$ and, as $F$ is a closed subgroup of the compact group $b X$, hence itself is compact, this residual subset is nonvoid. As the topology on $T$ is given by an invariant metric it follows that $\left\{\left.f_{n_{k}}\right|_{F}\right\}$ is equicontinuous on $F$. As $\rho$ is a continuous monomorphism of $X$ to $b X$ it follows that $\left\{\left.f_{n_{k}}\right|_{\rho^{-1}(F)}\right\}_{k}$ is equicontinuous. As $\rho^{-1}(F)$ clearly contains $D$, we have that $\left\{\left.f_{n_{k}}\right|_{D}\right\}_{k}$ is equicontinuous. Thus the statement $\left(^{*}\right)$ above has been proved. The proof of Theorem 1.1 is complete.

Proof of Theorem 1.2. Let $t_{1}, t_{2}$ be topologies on the abelian group $G$ such that $\left(G, t_{i}\right) i=1,2$ are topological groups that satisfy Pontryagin duality and have the same set $X$ of continuous characters.

It follows that $\left(G, t_{i}\right) i=1,2$ have the same Bohr compactification $b G$ and the canonical maps of $\left(G, t_{i}\right) i=1,2$ to $b G$ are the same map $\rho$. By Theorem 1.1 it follows that $\left(G, t_{i}\right) i=1,2$ have the same family of compact subsets. Now let $W$ be any closed limited neighbourhood of 1 in $T$. As $\left(G, t_{i}\right) i=1,2$ have the same set $X$ of 
continuous characters, they have the same family of $W$-character closed subsets (cf. Definition 2.1 above). By Proposition 2.2 as (G, $\left.t_{i}\right) i=1,2$ satisfy Pontryagin duality, they satisfy properties $P_{1}, P_{2}$ and $P_{3}$. Thus every $W$-character closed neighbourhood of the identity of $\left(G, t_{1}\right)$, because of the property $P_{2}$ of $\left(G, t_{2}\right)$ and because $\left(G, t_{1}\right)$ have the same family of compact subsets, will be a $W$-character closed neighbourhood of identity of $\left(G, t_{2}\right)$ and vice versa. Because $\left(G, t_{i}\right) i=1,2$ have property $P_{3}$, it will follow that they both have the same family of neighbourhoods of identity as neighbourhood bases. As they are both topological groups, it will follow that $t_{1}=$ $t_{2}$. This completes the proof of Theorem 1.2.

\section{REFERENCES}

1. H. H. Corson and I. Glicksberg, Compactness in $\operatorname{Hom}(G, H)$, Canad. J. Math., 22 (1970), 164-170.

2. E. Hewitt and K. A. Ross, Abstract Harmonic Analysis I, Springer-Verlag, Berlin, Gottingen, Heidelberg, New York 1963.

3. J. R. Hughes, Weak compactness and topological groups, Thesis, University of North Carolina at Chapel Hill, 1972.

4. - Compactness in locally compact groups, Bull. Amer. Math. Soc., 79 (1973), $122-123$.

5. I. Glicksberg, Uniform boundedness for groups, Canad. J. Math., 14 (1962), 269-276.

6. A. Grothendieck, Critères de campacité dans les espaces fonctionnels généraux, Amer. J. Math., 74 (1952), 168-186.

7. S. Kaplan, Extensions of Pontryagin duality I: Infinite products, Duke Math. J., 15 (1948), 649-658.

8. J. L. Kelley, General Topology, Van Nostrand, New York, N. Y. 1955.

9. J. L. Kelley and I. Namioka et. al., Linear topological spaces, University Series in Higher Math., Van Nostrand, Princeton, N. J., 1963.

10. I. Namioka, Separate continuity and joint continuity, Pacific J. Math., 51 (1974), $515-531$.

11. R. Venkataraman, Extensions of Pontryagin duality, Math. Zeit., (to appear).

Received December 13, 1974 and in revised form February 4, 1975. I thank the National Research Council of Canada for a Research Grant for carrying out this work.

UNIVERSITY OF MANITOBA 



\section{PACIFIC JOURNAL OF MATHEMATICS}

\section{EDITORS}

RICHARD ARENS (Managing Editor)

University of California

Los Angeles, California 90024

\section{J. DugundJI}

Department of Mathematics University of Southern California Los Angeles, California 90007

D. Gilbarg and J. Milgram

Stanford University

Stanford, California 94305
University of Washington Seattle, Washington 98105

\section{ASSOCIATE EDITORS}
E. F. BECKENBACH
B. H. NeumanN
F. WolF
K. YoShIDA

\section{SUPPORTING INSTITUTIONS}

\author{
UNIVERSITY OF SOUTHERN CALIFORNIA \\ STANFORD UNIVERSITY \\ UNIVERSITY OF TOKYO \\ UNIVERSITY OF UTAH \\ WASHINGTON STATE UNIVERSITY \\ UNIVERSITY OF WASHINGTON \\ $\stackrel{*}{*} \stackrel{*}{*} \stackrel{*}{ }{ }^{*}$ AMERICAN MATHEMATICAL SOCIETY
}

The Supporting Institutions listed above contribute to the cost of publication of this Journal, but they are not owners or publishers and have no responsibility for its content or policies.

Mathematical papers intended for publication in the Pacific Journal of Mathematics should be in typed form or offset-reproduced, (not dittoed), double spaced with large margins. Underline Greek letters in red, German in green, and script in blue. The first paragraph or two must be capable of being used separately as a synopsis of the entire paper. Items of the bibliography should not be cited there unless absolutely necessary, in which case they must be identified by author and Journal, rather than by item number. Manuscripts, in triplicate, may be sent to any one of the editors. Please classify according to the scheme of Math. Reviews, Index to Vol. 39. All other communications should be addressed to the managing editor, or Elaine Barth, University of California, Los Angeles, California, 90024.

The Pacific Journal of Mathematics expects the author's institution to pay page charges, and reserves the right to delay publication for nonpayment of charges in case of financial emergency.

100 reprints are provided free for each article, only if page charges have been substantially paid. Additional copies may be obtained at cost in multiples of 50 .

The Pacific Journal of Mathematics is issued monthly as of January 1966. Regular subscription rate: $\$ 72.00$ a year (6 Vols., 12 issues). Special rate: $\$ 36.00$ a year to individual members of supporting institutions.

Subscriptions, orders for back numbers, and changes of address should be sent to Pacific Journal of Mathematics, 103 Highland Boulevard, Berkeley, California, 94708.

\section{PUBLISHED BY PACIFIC JOURNAL OF MATHEMATICS, A NON-PROFIT CORPORATION}

Printed at Kokusai Bunken Insatsusha (International Academic Printing Co., Ltd.), 270, 3-chome Totsuka-cho, Shinjuku-ku, Tokyo 160, Japan.

\section{Copyright (C) 1975 by Pacific Journal of Mathematics} Manufactured and first issued in Japan 


\section{Pacific Journal of Mathematics}

\section{Vol. 57, No. $2 \quad$ February, 1975}

Norman Larrabee Alling, On Cauchy's theorem for real algebraic curves with boundary .......

Daniel D. Anderson, A remark on the lattice of ideals of a Prüfer domain ..................

Dennis Neal Barr and Peter D. Miletta, A necessary and sufficient condition for uniqueness of

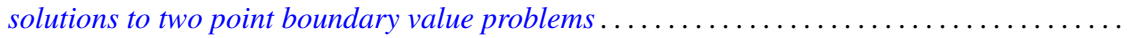

Ladislav Beran, On solvability of generalized orthomodular lattices . . . . . . . . . . ........

L. Carlitz, A three-term relation for some sums related to Dedekind sums . . . . . . . . . .....

Arthur Herbert Copeland, Jr. and Albert Oscar Shar, Images and pre-images of localization

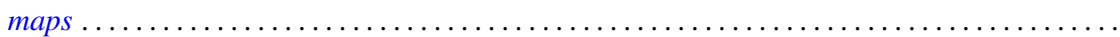

G. G. Dandapat, John L. Hunsucker and Carl Pomerance, Some new results on odd perfect

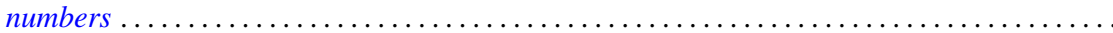

M. Edelstein and L. Keener, Characterizations of infinite-dimensional and nonreflexive

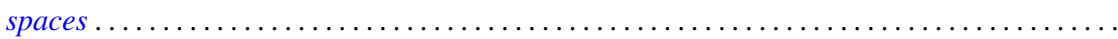

Francis James Flanigan, On Levi factors of derivation algebras and the radical embedding

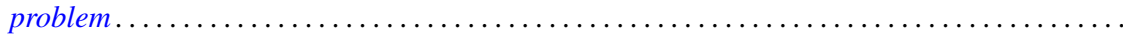

Harvey Friedman, Provable equality in primitive recursive arithmetic with and without

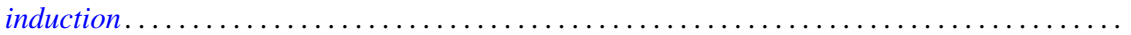

Joseph Braucher Fugate and Lee K. Mohler, The fixed point property for tree-like continua with

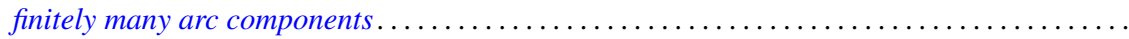

John Norman Ginsburg and Victor Harold Saks, Some applications of ultrafilters in

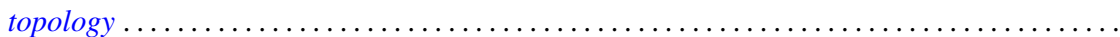

Arjun K. Gupta, Generalisation of a "square" functional equation .....................

Thomas Lee Hayden and Frank Jones Massey, Nonlinear holomorphic semigroups ..........

V. Kannan and Thekkedath Thrivikraman, Lattices of Hausdorff compactifications of a locally

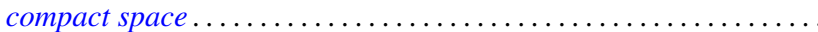

J. E. Kerlin and Wilfred Dennis Pepe, Norm decreasing homomorphisms between group

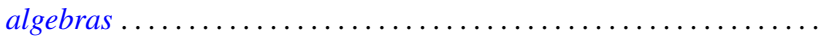

Young K. Kwon, Behavior of $\Phi$-bounded harmonic functions at the Wiener boundary ...

Richard Arthur Levaro, Projective quasi-coherent sheaves of modules .

Chung Lin, Rearranging Fourier transforms on groups...........................

David Lowell Lovelady, An asymptotic analysis of an odd order linear differential equation . . 4475

Jerry Malzan, On groups with a single involution .......................... 481

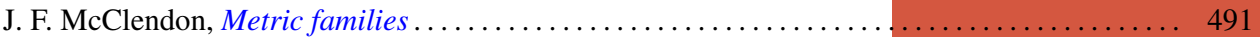

Carl Pomerance, On multiply perfect numbers with a special property .

Mohan S. Putcha and Adil Mohamed Yaqub, Polynomial constraints for finiteness of semisimple rings. .

Calvin R. Putnam, Hyponormal contractions and strong power convergence . . . . . . . . . 531

Douglas Conner Ravenel, Multiplicative operations in $\mathrm{BP} * \mathrm{BP} \ldots \ldots \ldots \ldots \ldots \ldots \ldots \ldots \ldots .539$

Judith Roitman, Attaining the spread at cardinals which are not strong limits . . . . . . . . . 545

Kazuyuki Saitô, Groups of *-automorphisms and invariant maps of von Neumann algebras . . . 553

Brian Kirkwood Schmidt, Homotopy invariance of contravariant functors acting on smooth

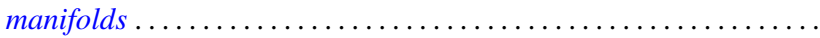

Kenneth Barry Stolarsky, The sum of the distances to $N$ points on a sphere.

Mark Lawrence Teply, Semiprime rings with the singular splitting property.

J. Pelham Thomas, Maximal connected Hausdorff spaces..............

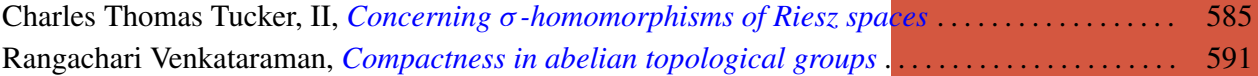

William Charles Waterhouse, Basically bounded functors and flat sheaves . . . . . . . . . . . 597

David Westreich, Bifurcation of operator equations with unbounded linearized part ......... 611

William Robin Zame, Extendibility, boundedness and sequential convergence in spaces of 